\title{
Civilisations
}

Revue internationale d'anthropologie et de sciences

humaines

59-2 | 2011

Les apparences de l'homme

\section{Esthétique et élaboration du féminin}

L'apport des cours de danse amateur

\section{Virginie Valentin}

\section{(2) OpenEdition}

1 Journals

\section{Édition électronique}

URL : http://journals.openedition.org/civilisations/2650

DOI : $10.4000 /$ civilisations. 2650

ISSN : 2032-0442

Éditeur

Institut de sociologie de l'Université Libre de Bruxelles

\section{Édition imprimée}

Date de publication : 30 juin 2011

Pagination : 125-143

ISBN : 2-87263-034-1

ISSN : 0009-8140

\section{Référence électronique}

Virginie Valentin, «Esthétique et élaboration du féminin », Civilisations [En ligne], 59-2 | 2011, mis en ligne le 01 juillet 2014, consulté le 19 avril 2019. URL : http://journals.openedition.org/ civilisations/2650 ; DOI : 10.4000/civilisations.2650

(c) Tous droits réservés 


\title{
Esthétique et élaboration du féminin \\ L'apport des cours de danse amateur
}

\author{
Virginie VALENTIN
}

\begin{abstract}
Résumé : Basé sur une enquête de terrain réalisée auprès d'une population féminine âgée de 4 à 60 ans, dans des écoles de danse amateur de la région parisienne et toulousaine, à travers l'exemple de la danse classique d'une part, et de la danse contemporaine et des danses "étrangères" de l'autre, cet article met en évidence comment l'esthétique que constituent gestuelles, costumes et parures forme le corps et y inscrit certaines valeurs propres à l'éthique de chaque âge. La danse classique, prime éducation des fillettes, consiste en l'apprentissage de la retenue et de la droiture, la danse contemporaine encourage une forme d' expression de soi ", les danses étrangères symbolisent des pouvoirs sexuels et génésiques propres à l'âge de femme. Ces capacités s'acquièrent autant par l'incorporation des gestuelles propres à ces danses que par l'usage des parures et costumes qui les accompagnent. Ces composantes sont en effet indissociables tant de la bonne réalisation de ces danses que de leur esthétique propre.
\end{abstract}

Mots-clés : féminité, danse amateur, parure, esthétique, corps.

Abstract: Drawing on fieldwork conducted within a feminine population aged from 4 to 60 years old, in amateurs' dance schools in Paris and Toulouse, this article highlights how aesthetic logics made by gestures, costumes and fineries shape the body in different ways in different dancing styles. Ballet dancing, as prime girlhood socialization, leads to learning of restraint and uprightness, contemporary dancing enables self-expression, while the "foreign" dances examined here symbolize the sexual and fertile powers of womanhood. These capabilities are acquired by both embodying the specific gestures of these different dances, and using costumes and fineries that come with it, two dimensions which are inseparable and both define the specificities of a dancing style.

Keywords: femininity, amateur dance, finery, aesthetics, body. 
$\mathrm{E}$ n France, quantité de fillettes prennent le chemin de l'école de danse classique de leur quartier ou de leur village. Dès lors, la pratique de cette technique chorégraphique et le modèle de la ballerine qui lui est lié occupent une place particulière dans la formation des femmes ${ }^{1}$. En analysant les livrets des grands ballets classiques qui ont diffusé la figure de la ballerine depuis plus de deux siècles, on a constaté ${ }^{2}$ l'aspect paradoxal de l'attachement des femmes à ce modèle car il s'agit, le plus souvent, d'un personnage de jeune fille et non de femme. Dans la mesure où cette image représente un parcours féminin inachevé, elle invite à se demander en quoi cette identification participe à l'élaboration de leur identité. Cela pose aussi une autre question : comment les filles dépassent ce modèle prégnant, c'est-à-dire quels sont les modèles qui s'y substituent et quelles sont les techniques chorégraphiques qui peuvent constituer un lieu de maturation identitaire, une fois abandonné l'investissement dans la danse classique ? Dans la mesure où la danse est une pratique artistique, la dimension esthétique y est centrale. La question est dès lors de saisir le sens des esthétiques corporelles valorisées dans la culture occidentale - et plus spécifiquement française - afin de comprendre dans quelle mesure elles contribuent à la formation du féminin.

Mon étude se fonde sur une ethnographie menée dans cinq écoles de danse parisiennes et toulousaines sur une période de trois ans ${ }^{3}$. Elle a consisté en l'observation des divers cours de danse qui y étaient proposés, des galas et de leurs répétitions ainsi qu'en une quarantaine d'entretiens avec des élèves âgées de quatre à soixante ans, de mères d'élèves et de professeurs. Par ailleurs, des entretiens avec vingt-cinq femmes qui ont pratiqué la danse ou la pratiquent encore, ont complété ces observations de terrain. Au cours de mes recherches, j'ai pu constater que, fascinée par l'image de la ballerine lorsqu'elles sont fillettes, la grande majorité des femmes débute par la danse classique puis interrompt cette pratique lors de l'adolescence, soit pour ne jamais reprendre une pratique chorégraphique, soit pour se tourner vers d'autres styles de danse. Ce passage se fait d'autant plus facilement qu'un important éventail de danse est généralement offert dans les écoles de danse amateur. Les statistiques confirment cela : $60 \%$ des filles qui ont pratiqué la danse classique l'ont délaissée au profit d'un autre genre ; la moitié se met alors à la danse jazz ${ }^{4}$. Il ressort également de mes enquêtes que les cours de jazz, de danse moderne mais aussi, aujourd'hui, de hip hop prennent le relais. Ces styles chorégraphiques sont proposés dans le cadre des écoles amateurs aux filles qui s'y adonnent volontiers durant l'adolescence. D'autres formes de danse constituent la dernière étape : danse contemporaine, d'une part, et danses orientale, flamenco, sévillane que j'appelle « danses étrangères », d'autre part. Si ces techniques chorégraphiques très diverses sont proposées, on constate cependant une certaine homogénéité dans la pratique de chaque classe d'âge : les styles chorégraphiques semblent scander les âges féminins, les symboliser, c'est-à-dire finalement en donner une acception sociale. Je me propose d'interroger ici

1. Sur la danse comme mode d'éducation corporelle « évidente » des filles, voir Rannou et Roharik $2006: 210$.

2. Sur l'analyse des livrets de ballets, le lecteur peut se reporter à un article antérieur où j’ai développé cet aspect (Valentin $2000: 95-98$ ).

3. Cette enquête a été menée dans le cadre d'une thèse de doctorat d'anthropologie (Valentin 2005).

4. Bulletin du département des études et de la prospective du Ministère de la Communication et de la Culture $\mathrm{n}^{\circ} 112$, juin 1996. Les chiffres plus récents de l'enquête de 2002 sont similaires (Notes statistiques $\mathrm{n}^{\circ} 10$, décembre 2004). 
cette correspondance établie entre styles de danse et classes d'âge. Dans la perspective de certains travaux sur l'éducation corporelle tels que ceux de Michel Foucault (1975), Georges Vigarello (1978) ou encore Pierre Legendre (1978), je tiendrai compte du fait que la danse est un lieu d'éducation et d'incorporation de valeurs, et je montrerai quelles sont les qualités développées et valorisées en fonction des âges et des styles chorégraphiques pratiqués. Ma réflexion se situe aussi dans la perspective des travaux en anthropologie de la figuration et de l'esthétique, notamment ceux d'André Leroi-Gourhan pour lequel le langage des formes est un « langage émotionnel dont une part des valeurs est d'origine biologique très générale et dont le code des symboles est au contraire fortement spécifié », au point qu'il « constitue proprement l'art figuratif» (Leroi-Gourhan 1964 : 207) ; de ceux sur l'ornement de Danièle Cohn, qui affirme que les arts sont soumis à un modèle narratif et, par conséquent, des arts du langage (Cohn 2008 : 92). Ces auteurs ont en effet montré que les arts seraient une forme de langage non verbal. À ces travaux s'ajoutent ceux sur le féminin de Véronique NahoumGrappe $(1988$; 1996) qui a souligné l'efficace non verbale de la perception visuelle de la forme humaine et de son esthétique. Dans la lignée de ces travaux d'anthropologie du corps, des arts, des formes et du féminin, je montrerai que, dans le cadre d'une pratique artistique telle que l'art chorégraphique, c'est à travers l'esthétique que des valeurs sont exprimées et transmises. Autrement dit, les diverses esthétiques chorégraphiques comportent un sens, une éthique ${ }^{5}$, et il s'agit de faire apparaître comment, en France, elles entrent en adéquation avec le processus de formation du féminin et son épanouissement. En suivant la dichotomie entre l'esthétique de la ligne et du rond soulignée par Véronique Nahoum-Grappe ${ }^{6}$ (1988), je me bornerai ici à exposer la technique corporelle qui constitue la prime éducation corporelle des fillettes pratiquant une forme de danse, à savoir la danse classique et, d'autre part, des danses auxquelles les femmes s'initient à l'âge adulte et qui les forme à une esthétique «autre » : danse contemporaine et certaines danses «étrangères ».

\section{De la droiture à l'éclat de la ballerine}

Les fillettes qui font leurs premiers pas dans un cours de danse classique apprennent essentiellement à savoir « se tenir » et se tenir droites. Elles incorporent ainsi la notion morale de droiture, et la « bonne tenue » que les fillettes doivent observer. Cette droiture, esthétique du redressement qui est aussi celle de la civilité et du pouvoir (Nahoum-Grappe 1988 : 17-20), est concrètement représentée par la «barre » à laquelle les élèves s'appuient pour effectuer leurs exercices. Les professeurs répètent souvent certaines corrections : « rentrer les fesses » ou «monter sous les fesses». Elles apprennent ainsi un certain maintien. L'insistance sur la tenue du bassin témoigne du fait qu'il s'agit d'apprendre à garder une certaine réserve. La recherche de l'étirement et de l'allongement du corps pendant le temps de « la barre » qui a lieu à chaque début de cours durant au moins une demi-heure, accompagne ainsi ce

\footnotetext{
5. Je parle d'éthique dans la mesure où le marquage des étapes de la maturation de la féminité constitue à la fois une reconnaissance et un respect de ce processus.

6. L'auteur souligne que deux esthétiques du corps coexistent dans l'histoire de la civilisation occidentale depuis le $\mathrm{XVI}^{\mathrm{e}}$ siècle : d'une part celle de la ligne qu'elle définit comme celle du temps socialisé, du redressement pédagogique mais aussi des positions sociales élevées qui est associée dans les représentations à l'homme, et d'autre part, celle du rond, de la courbe, associée dans les représentations scientifiques, artistiques et normatives à la femme.
} 
temps de la croissance. Les professeurs répètent en effet qu'il faut « se grandir », « s'étirer », «s'allonger». Cet allongement trouve son paroxysme à la fin de la barre où les filles, lorsqu'elles ont acquis la technique requise, font l'exercice du «pied dans la main » et du « grand écart ».

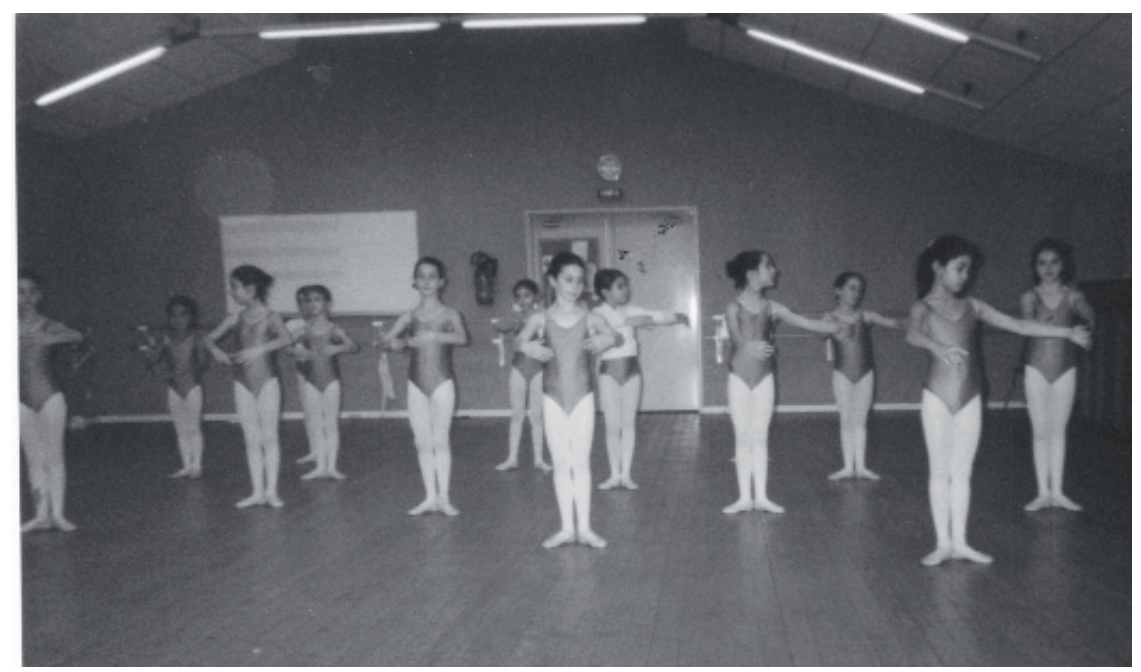

Figure 1

Fillettes prenant un cours de danse classique dans une école de quartier parisienne (février 2000).

(C) Photo Virginie Valentin.

La réserve des fillettes se dit aussi dans le langage vestimentaire puisqu'au cours de danse, elles doivent porter une tunique de couleur pâle, effacée et ramasser leurs cheveux en chignon - des signes conventionnels de réserve dans le monde social de la danse. De même, une fois qu'elles dansent dans l'espace de la salle, les pas qu'elles apprennent sont lents et liés : les corps sont policés. Les filles doivent aussi se tenir en lignes lors des exercices en salle puis lors des spectacles. Plus que de chercher à perfectionner la technique de chacune, les professeurs mettent beaucoup d'énergie durant les séances de répétition à essayer de les ranger en lignes et en colonnes. Toutes semblables car elles doivent effectuer les mêmes mouvements dans une synchronie parfaite, les filles doivent ainsi « faire corps $\rangle^{7}$ à l'instar des ballerines professionnelles, qui forment un corps de ballet autour de l'étoile. Cette « mise au pas » donne à voir un grand contrôle corporel. Si ces modalités de dressage des corps appartiennent en partie à l'orthopédie sociale que Michel Foucault a mise en évidence ${ }^{8}$, cette gestuelle commune est une préparation à une mise en scène des filles qui comporte une symbolique particulière, comme nous le verrons.

7. J'ai déjà eu l'occasion de mettre en évidence l'importance de l'uniformité du corps de ballet dans les grands ballets du répertoire classique. Cf. Valentin $2000: 98-100$.

8. Voir la troisième partie de l'ouvrage intitulée « Discipline » (1975: 159-264). 
Si droiture et réserve s'incorporent tout au long de l'apprentissage que les fillettes font de la danse classique, cette bonne éducation se concrétise généralement lors des spectacles de danse par l'incarnation de la figure de la ballerine blanche en tutu, le plus généralement long, et sur pointes, c'est-à-dire telle qu'elle est apparue dans les ballets romantiques du début $\mathrm{du} \mathrm{XIX}^{\mathrm{e}}$ siècle, ou «ballets blancs ». Cette figure emblématique de la danse classique est représentée par au moins une des classes d'âge, le plus souvent la plus âgée. C'est le cas dans un conservatoire de quartier parisien, où la danse des adolescentes de 12 à 15 ans, « la polka de Fanny Cerrito », référence explicite à une danseuse de l'époque romantique, est une mise en scène des adolescentes dans le pur style classique, sans narration, tel un tableau mettant en scène la maîtrise corporelle à travers l'exécution de gestes et figures de manière à la fois lente et liée, c'est-à-dire de ce qui est socialement reconnu comme la qualité propre à ce style de danse, la grâce. Dans une autre des écoles toulousaines où j'ai réalisé mon enquête, le rideau se lève sur le « ballet classique » où toutes les filles paraissent dans un costume blanc, une rose blanche dans les cheveux, évoluant en formant des lignes et des mouvements lents et retenus. Le ballet s'ouvre et se termine sur une pause regroupant toutes les classes d'âge réunies dans l'homogénéité d'un corps de ballet. L'école de danse se présente ainsi sous la forme d'une photographie des jeunes filles qui maîtrisent la technique et ont acquis la capacité d'exécuter des pas de danse dans un même rythme. Au cours des répétitions, la netteté des alignements et des mouvements d'ensemble a mobilisé toute l'attention des professeurs qui réitéraient leurs propos pour que les filles soient « en rythme », " ensemble » et qu'elles portent leur attention sur leur alignement. Ce corps de filles blanches évolue sur une musique lyrique. Les quatre saisons de Vivaldi où la musique est à la fois lyrique et rythmée est une oeuvre très prisée pour ce genre de mise en scène. Dans cet ensemble, on distingue une légère différence : certaines portent un tutu court, les plus âgées portent une tunique et une jupette nouée à la taille. Cette petite différence dans le costume est en réalité importante et les adolescentes y sont très sensibles. Dans le langage vestimentaire, elle sépare les deux classes d'âge, celle des 7-12 ans et celle des 13-18 ans. D'autres différences dans l'apparence marquent aussi les écarts entre les âges. Dans une autre école de danse, lors du gala un groupe de fille « fait les gitanes ». Ce groupe comporte des filles de 12 à 18, 19 ans. Il mélange ainsi au moins deux classes d'âges différentes. Celle des jeunes filles pré-pubères ou tout juste pubères et celle des jeunes filles menstruées, ceci s'ajoute au fait que les premières ne savent pas encore faire des pointes alors que les autres en portent. Interviewées à l'automne 2000, deux fillettes, élèves d'une école de danse parisienne, m'expliquent le plaisir qu'elles ont à interpréter ce rôle. Elles me décrivent les menus détails qui font l'intérêt de leur costume :

Maria : Là, on fait le rôle des gitanes, des gitans.

Louise: On aime bien la jupe.

Maria : Elle a plein de couleurs...

Louise : Plein de tissu...

Maria : J'aime bien le "V»là ! (au niveau du décolleté). Ce que j'aime beaucoup, c'est le foulard avec des petites médailles.

Louise : ça aussi j'aime, moi.

Maria : ça fait penser à une danse un peu gitane, j'aime bien. J'aime bien la ceinture parce qu'elle a une forme particulière avec comme une petite pointe ici et puis là, toute droite. 
Autant d'attributs qui soulignent une féminité qu'elles n'ont pas encore acquise : la parure dessine ainsi leur corps telle une promesse de leur physionomie future' ${ }^{9}$ D'autant qu'elles appartiennent, le temps de ce numéro, au " groupe des adolescentes ». Ici aussi un détail sépare néanmoins les plus jeunes des plus âgées : seules ces dernières portent le châle sans quoi l'on n'est pas une véritable « gitane » comme en témoignent également les propos de Maria et Louise :

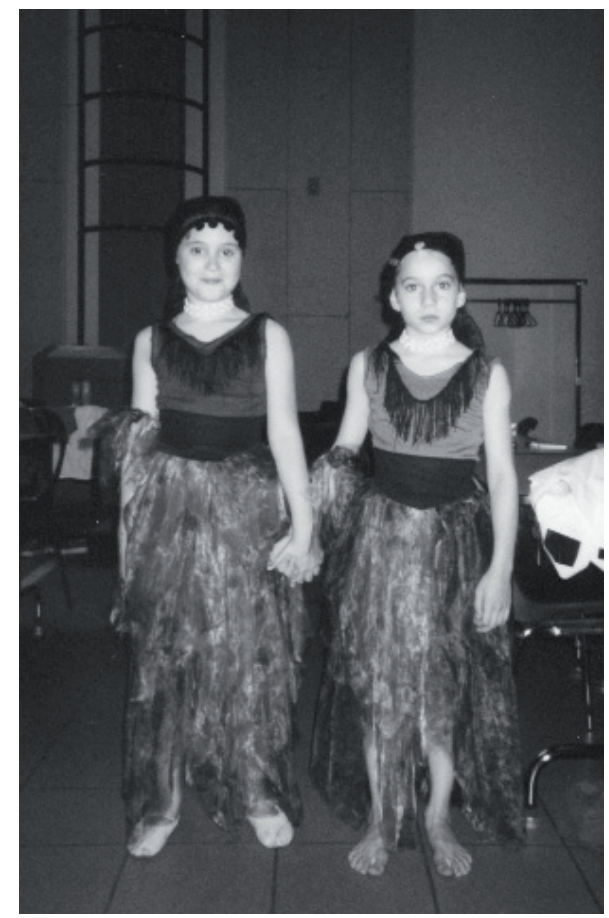

Figure 2

Maria et Louise dans le costume de gitane pour leur spectacle de danse classique de fin d'année.

(c) Photo Virginie Valentin.

Maria : Il y en a qui ont le même costume que nous.

Louise : Oui, mais elles ont des foulards pour danser, d'autres qui ont des foulards qui doivent s'attacher là [sur les hanches].

Maria : La différence, c'est pas le costume, c'est surtout ce qu'on sait faire. Elles font des choses plus importantes que nous : des portés ${ }^{10}$ des choses, comme ça. Donc elles sont un petit peu plus évoluées que nous, elles savent plus faire de choses que nous.

9. Jean-Claude Kaufmann a aussi remarqué l'importance de ce marquage symbolique dans la parure vestimentaire chez les préadolescentes : le maillot deux pièces permet de marquer et d'accentuer la différence sexuelle et de souligner la féminité (2010 : 92-93).

10. La fillette évoque le fait que les adolescentes s'initient, lors des cours d'adage, aux figures de couple où l'homme porte la femme. Ceci marque en effet le franchissement d'une étape dans l'apprentissage de la danse classique. 
Louise : Par exemple, les justaucorps : les grandes, elles ont des justaucorps rouges, bordeaux un petit peu, alors que nous, c'est marron. J'aime autant les deux mais c'est pas pareil.

La communauté de rôle entre filles pré-pubères et pubères, séparées durant l'apprentissage que les cours de danse constituent, est néanmoins très gratifiante pour les premières. Et le passage par un costume semblable témoigne ainsi d'une continuité dans la fabrique des filles nubiles.

Bien que l'école toulousaine de quartier déjà évoquée propose divers styles chorégraphiques qui sont représentés lors du gala (danse africaine, danse jazz, danse orientale), placer un ballet «classique » en début de spectacle est coutumier de cette école. Lorsque j’interroge le professeur de danse classique qui dirige également l'école, elle me répond «c'est notre image de marque ! » sans autre explication. Cette image renvoie à une autre, celle du défilé de l'Opéra de Paris ${ }^{11}$ et pose ainsi d'emblée le modèle et l'esthétique valorisée auxquels la danse classique aboutit : la jeune fille policée et élégante dans l'éclat d'une blancheur immaculée. C'est la même beauté que Jean-Luc Nancy a relevée dans un tableau d'Edouard Manet, La lecture. Le philosophe la qualifie comme la beauté « de l'éclatant, du blanc, de la séduction, de la femme ». Il précise : « Le tableau ne nous montre pas si l'homme est vraiment beau, il est dans l'obscurité mais aussi du côté du lisible. Le visible fait signe vers un sens qui est plus que du lisible. [...] Quelque chose est donné à travers la blancheur, l'éclat, la transparence, une tension vers la lumière » (Nancy 2009 : 27-28). Cette beauté du blanc éclatant associée au féminin (celui de la robe blanche de la jeune fille et des tissus qui occupe une grande partie du tableau) désignée ici comme « le visible» par opposition à la figure masculine, a un sens précis dans notre culture. Yvonne Verdier, étudiant les personnages des romans de Thomas Hardy, l'a remarqué : «Posant ainsi les femmes semblables les unes aux autres par le port d'un costume et d'accessoires identiques les jeunes filles forment un grand organisme relevant d'une même nature unique » (Verdier 1995 : 101). La mise en scène chorégraphiée des jeunes filles dans le ballet classique véhicule aussi cette symbolique particulière : elle représente le statut même de la jeune fille.

À l'adolescence, la pratique des pointes vient parfaire la ligne corporelle de la ballerine, sa silhouette gracieuse. C'est une expérience marquante qui occupe de ce fait une place particulière dans la biographie des femmes. Ceci s'explique par plusieurs facteurs. Ces attributs permettent aux adolescentes d'incarner dans la totalité de son image, ne serait-ce qu'un soir, ce modèle de féminité synonyme d'élégance et de beauté dans le monde occidental et par conséquent socialement valorisé. Ce sont aussi des chaussons qui blessent les pieds et peuvent faire surgir le sang ${ }^{12}$. Les filles retirent de cette expérience, qu'elles vivent comme une épreuve qualifiante, une certaine fierté. Une jeune femme me relate ainsi, enthousiaste

11. Le défilé de l'Opéra de Paris ouvre chaque année la saison théâtrale. L'ensemble de la troupe de l'Opéra, école de danse comprise, descend la scène du Palais Garnier dans le même costume, tutu court et blanc pour les femmes chemise et collant blanc pour les hommes.

12. Rappelons que dans certaines régions, comme la Bourgogne, traditionnellement «marquer» signifiait métaphoriquement avoir ses règles. Sur les différents sens de la «marque ». Cf. Verdier 1979 : 186-187. La douleur dans le marquage corporel transforme l'individu et témoigne de la douleur de l'épreuve aux yeux de la collectivité. Sur cette question, voir Houseman 1986 et Calderoli 1993 : 125. Cette dernière souligne que la valeur esthétique est parfois inextricablement liée à la valeur de l'épreuve douloureuse. 
et émue, les souvenirs liés à cette nouvelle technique où une tâche rouge était apparue sur le satin pâle de ses chaussons : «Je me souviens, j'ai saigné et j'étais très fière de cette tache de sang. J'ai gardé les chaussons. »

C'est aussi que cette expérience ambivalente, mélange de plaisir « narcissisant » et de douleur, située au début de l'adolescence et socialisée, entre en résonance avec leur puberté physiologique et, de ce fait, la symbolise. Elle est au cœur de l'identité féminine, dans la mesure où elle est, dans la pratique, un marqueur clair de la différence des sexes. La pratique des pointes est en effet strictement réservée à l'interprétation des rôles féminins et par conséquent, sauf rares exceptions - notamment dans des rôles parodiques où des hommes se travestissent -, aux femmes. S'ils magnifient le corps féminin, ces attributs le rendent aussi vulnérable et une nécessité de soin en découle. Les filles sont obligées d'utiliser pansements et coton cardé (coton chirurgical), seul matériau qui évite l'échauffement des doigts de pieds dans les pointes. Tout un vocabulaire du soin entoure cette pratique : ce qu'elles appellent des « poupées » naissent ainsi au bout de leurs pieds. Jeu métaphorique autour de la fécondité et de la maternité.

Aussi ces chaussons fascinent-ils les petites filles et certaines désirent « savoir ce que ça fait » et essaient parfois de faire des pointes avant d'y être autorisées. Elles déploient pour cela des trésors d'imagination, montant sur les chaussons de fortune que constituent les espadrilles ou subtilisant dans la malle commune de l'école de danse une paire de chaussons, comme deux fillettes de 9 ans me le racontent :
Moi j'ai essayé avec celles-là. Elles sont au ballet. On a essayé pour voir comment ça faisait. Ça fait mal, pas trop mais quand tu restes trop longtemps...
Ma sœur fait de la danse aussi, elle fait du classique et du jazz aussi. Elle prend des pointes pour le classique ça fait très mal. Elle m'a dit que ça faisait très mal. Des fois quand elle en mettait, elle saignait.

En fait, cette volonté d'appropriation que manifestent les fillettes et qui est due au secret et à l'interdit posé par les professeurs, témoigne de l'importance initiatique de ce passage ${ }^{13}$.

$\mathrm{Si}$ les filles désirent faire l'apprentissage de cette technique douloureuse c'est essentiellement parce qu'elle permet de parfaire l'apparence et la beauté de la ballerine. Et l'attrait de ces attributs spécifiques qui permettront de se tenir sur la pointe des pieds, chose « magique », est lié tant à cette utilisation énigmatique du corps dans son ensemble qu'à l'esthétique inhérente aux chaussons, comme en témoigne une jeune femme : « Je supportais, je supportais » souligne t-elle. Elle enchaîne comme si c'était ce qui permettait précisément de supporter la douleur : « Je me regardais dans la glace ». Je lui demande si elle trouve cela joli : « Oui, il y a un côté esthétique, le satiné, finalement c'est dans une matière plus jolie que les chaussons de demi-pointes : c'est brillant! » (entretien, novembre 2003). Cet attrait de l'aspect soyeux n'est pas propre à notre culture, par exemple, Monique Jeudy-Ballini a souligné combien il était important dans la danse des masques, chez les Sulka de Nouvelle-

13. Patrizia Ciambelli a mis en évidence ce phénomène pour le maniement des boucles d'oreilles qui, implique une légère modification corporelle comme dans les épreuves initiatiques ou l'on apprend à maîtriser ses émotions, à supporter la douleur. «La transformation du corps féminin se dit et se fait aussi au moyen de la pratique du perçage et de la parure en autant d'étapes qui correspondent à des âges clés de la vie d'une jeune femme et visent à assurer et à faire croître sa fertilité, cette dernière étant indissociable de la beauté féminine ». (Cf. Ciambelli $2002: 14)$ 
Guinée, où la beauté se dit en termes de brillance, d'éclat, de clarté ou encore de luisance (Jeudy-Ballini 1999: 10). Notons que ce sens est contenu dans le terme d' «étoile » qui qualifie les danseuses ayant gravi les différents échelons du corps de ballet de l'Opéra de Paris.

Ainsi la complexité des facteurs qui cristallisent autour des chaussons de pointe une certaine aura font de l'accès à ceux-ci un marqueur essentiel d'une véritable puberté sociale au sens où Arnold Van Gennep l'entendait. Mais parce qu'elle est une expérience douloureuse ${ }^{14}$ t qu'elles «savent» dorénavant « ce que ça fait», celles qui pratiquent la danse classique en amateur se détournent généralement de l'esthétique de la danseuse classique, après un passage qui n'aura duré, pour bon nombre d'entre elles, que quelques mois et le temps d'un gala de fin d'année.

\section{S'exprimer}

Nombreuses sont les d'adolescentes qui trouvent la danse contemporaine opaque, difficile à appréhender. Sans doute est-ce dû à la mise en avant de l'individualisation, du rapport plus personnel à la danse que cette technique corporelle propose, auquel la majorité des adolescentes n'est pas encore prête à s'engager et/ou qu'elles découvrent. Une jeune femme de 32 ans, Élodie m'expliquait qu'ainsi elle ne pouvait répondre à la demande qui lui était faite dans ce cours :

La contemporaine, je me souviens que je n'avais pas compris. J'étais ado, je n'avais rien compris. Je me demandais : "Qu'est-ce qu'il attend de nous? " Et je me disais : "C'est n'importe quoi ». En fait, je voyais bien ce qu'il attendait de nous mais je ne me sentais pas capable.

Je lui demande alors de préciser :

Laisser tomber nos références, nos petits cadres qui nous protégeaient bien, pour essayer de danser autrement, pour nous laisser plus aller à l'improvisation, ce qui à quinze ans me foutait une trouille bleue. Parce que j'avais besoin de repères. Oui, ça me protégeait bien. Donc je me souviens du stage de contemporaine comme d'un échec. (entretien, avril 2003)

Incompréhension ou refus de se défaire de ses habitudes pour aborder une forme de danse qui demande de puiser dans des ressources plus personnelles? La danse contemporaine engage la subjectivité de la personne et amène à s'explorer soi-même. Une plongée intérieure que toutes ne sont pas prêtes à faire. Quoi qu'il en soit, une dizaine d'années passeront avant qu'Élodie aborde à nouveau la danse contemporaine et puisse prendre plaisir à cette technique corporelle plus introspective :

Le contemporain, c'est des cours où tu te mets beaucoup en question en tant que personne à travers ce que tu danses, la manière dont tu t'exprimes, ce que tu montres aux autres... Comme c'était une question qui me préoccupait beaucoup à ce momentlà. Faire ces cours-là, y aller, c'était une épreuve. De savoir ce que j'allais montrer de

14. Selon Arnold Van Gennep, les marquages corporels ont une fonction d'agrégation à un groupe déterminé (Van Gennep 1909 : 103). Prenant l'exemple d'une cérémonie de circoncision des garçons Tsonga, Victor Turner (1991 : 64) montre aussi l'importance de ces marquages et des épreuves douloureuses dans le changement de statut social. 
moi aux autres, à travers la manière dont je dansais. Et il y avait des moments où je n'y arrivais plus, toujours avec le prétexte "j'ai oublié les pas ». Et ça, j'ai toujours fait. (entretien, avril 2003)

Cette forme de danse correspond ainsi parfois pour les jeunes femmes à l'approfondissement d'une recherche par rapport à leur image qu'il s'agit de dépasser car c'est aussi une recherche de sensations corporelles internes et fines, de connaissance de leur corps. Pour certaines la pratique de l'improvisation qui fait partie de l'apprentissage est ce moyen de « se découvrir », de «se trouver». Chloé, jeune femme de 28 ans a ressenti cette danse comme un moyen d'expression intense :

La danse contemporaine, quand-même, au départ, m'a posé question : parce qu'arrivé à dix-sept ans, on te demande de rouler au sol, de ramper. Je me rappelle au premier cours je me suis dit « mais qu'est ce que c'est que ça? " Cette prof travaille beaucoup sur l'improvisation dansée. C'était une période où je me cherchais, $j$ 'ai vite pris plaisir à danser à travers ces mouvements proposés, ces mises en situations, à travers ces improvisations, c'était une manière pour moi de m'exprimer. Je pense que c'était vraiment une rencontre avec moi-même. C'est ce qui m'a donné envie de continuer, j'ai ressenti le bien être que ça pouvait apporter. Je pense que c'est vraiment ce qui m'a donné envie de poursuivre. (entretien, avril 2003)

Cette technique de danse qui explore le champ des possibles du corps en mouvement est ainsi vécue comme une danse de découverte de soi. Pour ces jeunes femmes, danser sans critère particulier permet d'exprimer sa subjectivité, sa manière propre d'appréhender corporellement le monde. Analysant les modalités d'apprentissage de la danse classique et de la danse contemporaine, qualifiant l'une de régime de discipline et l'autre de régime de singularité, Sylvia Faure a souligné le travail d'invention et de personnification, lié à l'absence de modèle, propre à la danse contemporaine (2000 : 115-119). Dans une telle configuration, le dévoilement de la « personnalité » ne semble pouvoir être que le fait de jeunes filles ayant atteint une certaine sécurité par rapport à leur image corporelle.

Les professeurs demandent à leurs élèves de porter une attention particulière à l'ensemble des points de leur corps, de «visualiser» des trajectoires de mouvements. Ceci demande une capacité d'abstraction que toutes ne cherchent pas à développer. Pourtant, le travail de contraction-release élaboré par Martha Graham et utilisé de manière «vulgarisée » lors des échauffements dans la plupart des cours de danse contemporaine apparaît comme une autre étape de la libération du bassin. Traduit en français par « contractions », ce terme peut évoquer les mouvements du corps de la femme lors de l'accouchement. Le cours de danse contemporaine peut être le lieu d'appropriation de certaines propriétés de leur corps dont les jeunes filles n'ont pas encore fait l'expérience. Sans rechercher une forme définie et préétablie, une esthétique particulière, à l'instar des avant-gardes artistiques qui ne recherchent pas l'harmonie mais visent davantage la rupture avec un schéma perceptif institutionnalisé (Eco 2007 : 378), cette technique fait naître de nouvelles sensations corporelles. C'est ce que constate Chloé qui reprend l'apprentissage de la danse contemporaine à vingt-cinq ans. Ayant quitté son ami avec lequel elle vivait depuis six ans, la jeune femme s'était réinstallée seule. Elle réalise qu'elle n'a pas fréquenté les écoles de danse par hasard cette année-là :

Je me reconstruisais, j'ai fait vachement de danse pour quelqu'un qui n'en a pas fait pendant cinq ans. Je n'avais jamais fait le lien! Après, je n'étais plus toute seule dans la vie. Je suis partie du centre ville, donc je n'ai plus eu d'énergie pour prendre des 
cours et six mois plus tard, j'étais enceinte et j'ai fait du yoga et depuis je n'ai plus repris la danse. (entretien, avril 2003)

S'initier à la danse contemporaine a ainsi correspondu à une forme de « recentrage sur elle-même » tant dans la réalité de sa vie privée que dans la prise en compte de sa dimension corporelle. Grâce à ces cours qui commençaient par des massages des pieds, des exercices de relaxation et continuaient par une technique de danse issue des arts martiaux « dans le sol» et « ronde », elle pouvait développer des sensations fines, une manière d'appréhender son corps en douceur et en profondeur. Ceci se prolongea lorsqu'elle fut enceinte de son premier enfant. Elle fit alors du yoga, technique corporelle de relaxation, d'écoute du corps. Elle pratiqua alors la danse contemporaine dans un but précis : "C'était aussi une façon de me faire du bien ». Cette pratique représente pour elle un soin du corps important.

Proposant un dépassement de l'image afin de faire émerger une expression personnelle issue d'un ressenti et des émotions, la danse contemporaine occupe ainsi une place particulière : elle permet de ressentir véritablement son corps, d'en faire émerger les potentialités et de pouvoir le modeler à l'image de son désir. L'anthropologue André Leroi-Gourhan affirmait que les arts prennent leur source dans la sensibilité viscérale. À travers cette technique corporelle, les femmes peuvent utiliser ce qu'il a nommé "l'appareil esthétique $»^{15}$ pour exprimer leurs désirs intimes. La danse contemporaine, parce qu'elle laisse une grande place à l'expérimentation, permet cette expression fine et profonde du corps humain.

On préfèrera l'expression de «danses étrangères » à celle de «danses du monde » communément utilisée pour qualifier les danses issues d'autres cultures auxquelles nombre de femmes s'initient à l'abord de la maturité ${ }^{16}$, car dans cette formulation est contenue l'idée d'étrangeté qui préside aussi à la transformation du corps féminin : le corps devient autre et c'est en s'appropriant cette altérité qu'elles deviennent femmes. Comme Véronique NahoumGrappe l'a développé, dans notre culture, dans les arts visuels, une forme, complément de la ligne correspond à ce phénomène féminin, la rondeur : «l'esthétique de l'arrondi intervient dans la niche écologique de la Belle Femme» (1988: 24). Cette esthétique se décline différemment en fonction des techniques chorégraphiques proposées. Voyons comment.

\section{«Danser le couple "}

L'apprentissage de la danse sévillane fait entrer celle qui la pratique dans un rapport radicalement autre à son corps en la mettant en relation avec des rondeurs et courbes d'ordre symbolique ainsi que par le fait qu'il s'agit d'une danse à deux. Ces deux aspects s'associent logiquement, peut-on dire, dans la mesure où la rondeur corporelle est le signe de l'entrée potentielle dans la sexualité. Jean-Claude Kaufmann le note dans sa Sociologie du couple, l'entrée dans le rapport de couple change la donne du rapport à soi, elle en est un élargissement. L'individu passe du je au nous. Pour le sociologue, « l'amour fondateur du lien conjugal est une dénégation de ce je au nom du tu et du nous » (1993: 72). Même s'il ne s'agit pas d'un couple réel, la sévillane est une danse à deux dont la gestuelle, les figures engagent dans un

15. C'est ainsi qu'André Leroi-Gourhan qualifie le langage des formes permettant aux « figurants » et aux « spectateurs » de partager des valeurs communes (Leroi-Gourhan 1964 : 207-208).

16. Ces danses sont abordées à partir de 18 ans environ, dès que les jeunes filles assument le changement corporel de la puberté mais cela peut être beaucoup plus tardivement en fonction des femmes et de leur rapport au corps. 
rapport particulier à l'autre. Elle constitue ainsi une forme d'initiation au rapport de couple. Mais quel type de « nous » peut alors émerger dans cette mise en scène chorégraphique ?

Tout d'abord, l'avantage sur la vie réelle est que les femmes peuvent s'initier à ce type de rapport en dansant avec d'autres femmes car, à la différence d'autres danses de couple, les hommes pratiquent peu la sévillane. Une approche intéressante pour certaines femmes qui ont reçu une éducation bourgeoise et rigoriste, pour qui le rapport à l'autre sexe se vit comme une transgression. Bien que ce soit essentiellement des femmes âgées de dix-huit à cinquante ans que l'on trouve dans les cours, la sévillane est une danse du « courtisement » : traditionnellement elle est apprise aux jeunes filles en Espagne et elle est souvent vécue comme une initiation au flamenco. De manière plus générale, c'est par là au rapport de séduction que les femmes s'initient.

Étudions donc la structure de cette danse. La figure principale en est la «passade » qui s'exécute dans un échange avec un partenaire : une jambe se lève, pliée vers l'autre, puis la danseuse passe de l'autre côté, échangeant sa place avec lui. La passade est l'aventure amoureuse de courte durée, un diminutif de la passion, selon la définition du dictionnaire. Dans cette danse composée de multiples passades, les femmes décrivent des courbes au sol autour de leur partenaire, elles le charment aussi en dessinant des volutes avec leurs bras et leurs mains : balancement des corps, balancement des cœurs. Tout un lexique gestuel du rond, du courbe, s'incorpore. Les passades prennent cependant des colorations différentes selon les sévillanes, qui sont au nombre de quatre. Comme différentes élèves me l'expliquent, chacune décrit un moment du rapport amoureux : la rencontre, la séduction, la dispute et la réconciliation. Christophe Apprill a étudié les danses de couple, et en particulier les diverses manifestations et représentations qui se cristallisent dans le tango : « Des scansions traditionnelles de la vie de couple, les représentations traditionnelles [du tango] ne retiennent que la rencontre et la fusion » (Apprill 2008 : 45). À la différence de cette vision exaltée qui préside au tango, la sévillane est la métaphore du cycle existant dans toute relation de couple, avec ses hauts et ses bas, la «tendre guerre » inhérente à toute relation intersubjective qui s'illustre dans cette danse par quatre temps forts. C'est donc un apprentissage symbolique du rapport de séduction, le code d'un courtisement ${ }^{17}$ qui durerait tout au long d'une relation. Il s'agit sans doute d'une manière de magnifier, de sublimer la réalité prosaïque par une poétique dansée du couple et d'y faire entrer les dissensions liées à l'insatisfaction qui ne manque pas de survenir dans toute vie de couple (Kaufmann 1993 : 102-108). La sévillane suggère en effet une mise en scène particulière de ce processus. Ainsi faut-il maîtriser ses différentes séquences, scène de séduction ou violent face à face toujours dans un rapport distancié à l'autre. En effet, le rapport qu'entretiennent les danseurs est distancié, puisque s'ils sont l'un en face de l'autre, jamais ils ne se touchent : la rondeur de la sévillane est symbolique. Esthétique du désir, elle s'inscrit dans les figures, les gestes et, on le verra, dans la parure et la vêture féminine. Comme cela m'est précisé par une élève, la séduction passe aussi par l'intensité du regard des deux danseurs, la femme ne s'abandonnant dans les bras de son danseur qu'à la fin de la danse, dans le dernier geste du bien parado :

17. La sévillane, danse andalouse comme son nom l'indique, est issue de l'école bolera qui a corrigé dans un sens à la fois baroque, romantique et bourgeois l'ensemble des danses populaires espagnoles. (Leblon 1995 : 76) 
Il fallait y aller: il y a le regard de l'autre. Quand tu danses tu es dans le regard de l'autre. Quand tu tournes après, il faut aller chercher le regard de l'autre. En même temps il n'y a pas le contact qu'il y a dans le tango. C'est vraiment dire quelque chose à l'autre. (entretien, décembre 2003)

Si dans le tango, le rapport homme-femme est dissymétrique, la femme étant plutôt offerte et énergique, l'homme plus retenu et grave (Apprill 2008 : 51), l'un et l'autre ont une gestuelle davantage similaire, bien que l'idée de force et de retenue pour l'homme existe aussi dans le style flamenco (pour la description duquel on a parfois eu recours à la métaphore du taureau, voir Leblon 1995). Mais ce sont surtout la mise (robe ornée pour la femme, pantalon et sobriété pour l'homme) et la parure qui les différencient (Leblon 1995 : 76). Dans cette chorégraphie subtilement réglée, les femmes affichent tous les attributs et ornements qui « font» la femme sensuelle. En particulier la vivacité des couleurs de la robe, la large rose rouge qu'elles piquent dans leur chignon lors du spectacle exacerbe cette qualité. À l'Académie toulousaine où Karine danse, la robe est de couleur « sang et or », m'expliquet-elle, et le maquillage que la jeune femme juge « outrancier» dessine son visage :

Des petites boucles d'oreilles ou des créoles, un rouge à lèvre très très fort les yeux noirs et une fleur dans les cheveux. Beaucoup de blush. À la répétition, elle [la professeure] a montré sur elle. Elle n'a pas précisé pour le vernis à ongles: certaines en avaient, moi non. Elle insiste sur le tour de l'œil très charbonneux, la bouche très rouge, les pommettes très roses. (entretien, juin 2003)

Elles sont ainsi clairement placées du côté du rapport de séduction. Monique JeudyBallini a souligné le choc émotionnel propre au pouvoir attractif de la couleur et le fait que ce pouvoir esthétique résidait dans son impact visuel (2006 : 5-20). En France aussi, cet impact joue. Si la beauté et l'émotion esthétique étaient privilégiées dans la danse classique, c'est l'émotion sensuelle qui est en jeu à travers ces couleurs vives et prononcées. Mais les femmes se doivent de garder une certaine tenue : corps hiératique, cheveux tirés en chignon tout comme dans la danse classique. La rondeur de leurs mouvements et des larges anneaux qu'elles portent aux oreilles, les «créoles », ces boucles d'oreilles volumineuses au nom exotique, affichent une certaine disponibilité sexuelle, certes, mais tout en observant toujours une grande retenue : le buste et le corps dans son ensemble restent droits. Pour le spectacle, le professeur a demandé que les cheveux soient «très tirés : ni bijoux, ni alliance ». Ces similitudes avec la danse académique montrent que la danseuse espagnole est également une femme qui « sait se tenir ».

Dans la sévillane, les rondeurs sont aussi représentées sous la forme des fameux pois de la robe de flamenco et dans les balancements de la robe à volants. Les femmes aiment ces robes que l'on retrouve dans le prêt-à-porter. Certaines qui ont des difficultés à exprimer leur féminité dans la vie courante apprécient particulièrement de la porter. C'est le cas de Jeanne, 45 ans, fille d'exilés espagnols qui s'initie au flamenco depuis quelques années, renouant ainsi avec une image féminine enfouie :

J'ai une photo de moi et de mes sæurs, de ma mère mince avec une robe à pois et on avait la même robe rouge à pois blanc avec le petit cloquet, là. On en était folles! J'en ai une passion! Et d'ailleurs j'ai acheté une robe avec des petites fraises, des petites cerises et c'est comme les pois. (entretien, décembre 2003) 
Pour elle, la robe à pois évoque les fruits rouges dont les femmes enceintes raffolent. Les dessins ronds apparaissent ainsi comme une symbolisation du « fruit ». Parées de cette robe-fruitière, les jeunes femmes paraissent tout à la fois en possession de leurs pouvoirs de séduction et de fécondité. Aussi la sévillane est une danse terrienne, elle ancre les filles au sol ${ }^{18}$. Un enracinement qui se signale par le port de souliers à talons utilisés pour se faire entendre. Les professeurs insistent généralement pour que les filles tapent fort. Ainsi dans l'académie toulousaine où Karine danse, le professeur assène sans relâche aux femmes : « Frappez fort. Affirmez-vous ! (observation participante, décembre 2003). Si elles portent des chaussures de « caractère ", c'est pour affirmer le leur : la danse flamenca est avant tout une question de tempérament (Leblon 1995 : 83). Mais ce martèlement ne se fait pas n'importe comment, encore faut-il qu'elles tapent ensemble, c'est-à-dire en rythme : « La prof dit qu'on ne peut pas être une danseuse solitaire, on doit être sur la même ligne et en rythme » m'explique la jeune femme (entretien, juin 2004). La communion rythmique et sonore accorde ainsi les corps et leur restitue ou affirme leur part charnelle. La technique chorégraphique de la sévillane donne un cadre, un rythme, une coloration au temps féminin en le socialisant et en l'inscrivant dans autre cycle, celui du couple.

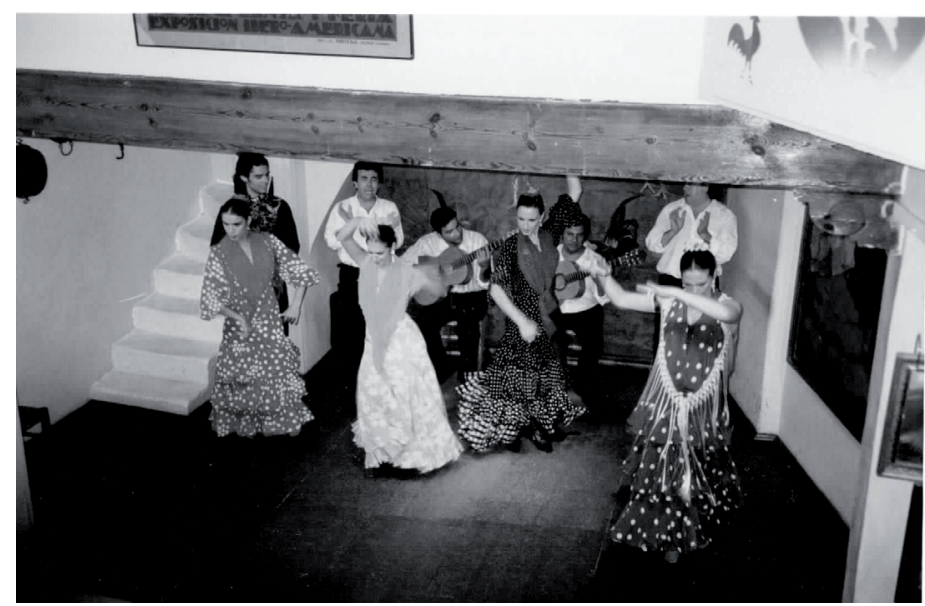

Figure 3

Femmes portant le costume flamenca utilisé dans la sévillane: robe à pois, châle, rose rouge et chaussures de caractère.

(C) Photo Virginie Valentin.

\section{Une culture féminine : la femme-potière}

La rondeur de la danse orientale s'inscrit plus profondément dans les corps. Aurélie, jeune femme, élève d'un cours de danse orientale toulousain, admirant son professeur de danse, une femme bien en chair, s'exclame ainsi : «Elle est belle ! Je suis contente d'avoir pu la voir danser, elle est gracieuse, ça lui colle complètement à la peau » (entretien, avril 2004). À ce

18. Pour les Gitans d'Andalousie, la femme est solidement accrochée à la terre (cf. Pasqualino 1998 : 111). 
cours, l'arrondi s'incarne d'abord sous les traits d'un modèle de femme, en l'occurrence le professeur, avant de s'incorporer grâce aux consignes de celui-ci.

Lors des premiers cours de danse, ce professeur utilise des métaphores en rapport avec la culture de la terre pour transmettre aux femmes la manière dont elles doivent ressentir leur corps comme s'il s'agissait de reprendre contact avec les forces terriennes : « Là ! Vous êtes dans la position de la paysanne. C'est idéal pour cultiver les champs. C'est une bonne position ça. Si vous êtes bien, restez sur les talons. » (observation participante, Janvier 2004) Elle montre aux femmes comment se planter dans le sol : à plusieurs reprises, elle laisse retomber lourdement une jambe et le pied contre le sol en ajoutant : « il faut avoir l'impression de porter une jarre sur la tête ». Elle souligne également que l'on peut ressentir des « sensations nouvelles ». Tout en donnant ces indications, elle engage les femmes à faire tourner leur bassin autour d'elles-mêmes, telles des potières façonnant leur matière propre. Cette séquence dure de longues minutes. Puis : « Il faut que les aines s'ouvrent comme des petites fleurs $»$. Cette mise en train est destinée à changer radicalement la manière de ressentir son corps. Ici, point d'homme : le professeur distille un discours sur une culture féminine. Une élève me narre :

Je trouve que c'est surtout le costume qui y fait beaucoup. Et dès qu'on a le costume, ça change, parce qu'on ne voit pas les jambes et qu'on doit beaucoup bouger par les jambes. Quand on a une jupe, on a l'impression, vraiment, qu'il n'y a que ça qui bouge, alors qu'en pantalon, on voit les jambes bouger. Je regarde dans la glace je me dis: «Non ». Elle me dit : "Mais si, c'est ça! » Je dis : «Non, non, non! » Alors que quand on a la jupe on ne voit rien: on ne voit que les hanches bouger. Et c'est ça! (entretien, février 2004)

Cette jeune fille de dix-neuf ans s'étonne de voir que le mouvement d'ensemble du corps est impulsé uniquement par les jambes que la jupe cache. Car c'est bien là le secret de cette danse. Secret que le professeur livre lors des premiers cours en insistant comme si les femmes n'allaient pas l'entendre: "On croit que ce sont les fesses qui bougent mais ce sont les genoux qui font tout, elles ne se promènent pas toutes seules les fesses, elles sont rattachées au reste du corps, donc en pliant le genou, on se déhanche. C'est anatomique ! ». (observation participante, juillet 2003). Les jambes se plient et se tendent comme des pistons, c'est ce mouvement que les femmes doivent incorporer pour que la jupe ondule, comme il se doit. Pour accentuer l'effet du mouvement, on noue un foulard autour des hanches. Il souligne les circonvolutions du bassin et les vibrations du ventre. Cet accessoire est indispensable à l'exécution de la danse. Dans les mariages maghrébins aussi les filles ne peuvent danser que munies d'un foulard. Ces danses sont destinées à éveiller la sensualité et à les préparer à la défloration ${ }^{19}$. Objet de la transmission matrilinéaire dans ce cadre, le foulard contribue à la bonne réalisation de la danse dans le rite comme dans l'apprentissage pédagogique.

C'est sans doute parce que ces mouvements sont particulièrement sensuels et mettent en évidence les potentialités fécondes des femmes que certaines sont gênées, voire confuses, lorsqu'elles en parlent. Elles ont en effet parfois des difficultés à expliquer pourquoi elles pratiquent cette technique. Ainsi une jeune femme qui s'y initie depuis peu au sein de la même école de quartier toulousaine :

19. Noria Boukhobza a montré cela dans son ouvrage sur les femmes algériennes en France (2002: 124). Sur la préfiguration de l'acte sexuel dans les mariages tunisiens (cf. Nicolas $2000: 54-55$ ). 


\begin{abstract}
Il y a des mouvements qu'on n'ose pas forcément faire. Oui, moi, au début, j'étais gênée. Pour les accents avec le buste, la poitrine. C'est pas facile la danse orientale, il faut vraiment savoir dissocier le buste du bassin, des fesses. Soit on bouge que le bassin et dans ce cas là, il faut rester immobile, soit que les hanches. (entretien, mars 2004)
\end{abstract}

Comme dans les danses modernes, les mouvements d'isolation mettent particulièrement en valeur les parties « sexualisées» du corps. Comme la pratique de la danse classique en témoigne et comme certains sociologues l'ont souligné20 ${ }^{2}$ l'éducation des filles ne favorise pas toujours la libération de ce type de mouvements et de sensations. Ces parties du corps sont en effet largement contrôlées socialement. Aussi la difficulté réside-t-elle ici surtout dans le fait de ne mobiliser que les muscles nécessaires pour bouger seulement ces parties-là. La danse orientale requiert une grande sobriété dans la manière de se mouvoir. Aucun mouvement ne vient s'ajouter à cette mise en valeur de la puissance sexuelle des femmes. Ceci demande peu d'énergie mais pourtant elles ressortent de certains cours avec des courbatures aux hanches et au ventre. C'est que cette danse du ventre, comme on l'appelle communément, doit détendre cette partie du corps, les courbatures venant, paradoxalement, de l'effort pour obtenir cette relaxation des parties habituellement retenues. Une autre jeune femme interrogée en même temps que la précédente : «C'est super précis. Les doum, les tac... on plie on tend on plie on tend... Ça n'a l'air de rien mais ça donne des courbatures sous les hanches et le ventre » (entretien, février 2004). Les femmes s'appliquent ainsi à intégrer ce savoir-faire constitué de quelques mouvements bien maîtrisés. Martine, une dentiste de quarante-huit ans, nouvellement entrée dans ce cours, affirme que la danse orientale lui a libéré le corps. Elle a aussi remarqué que cette pratique modifie la démarche et le corps lui-même : « Je vois les filles, après quelques mois, elles sont plus rondes, elles ne marchent pas pareil dans la rue. Elles ne sont pas droites comme d'autres... » (entretien, janvier 2004). Ainsi, la danse orientale façonne le corps en l'arrondissant et en le creusant. Tout dans la gestuelle de cette danse contribue à la mise en valeur du lieu matriciel, qui est ainsi une forme de célébration de la fécondité féminine. Certaines femmes m'ont ainsi confié qu'elles étaient tombées enceintes et avaient eu un enfant peu après l'entrée dans l'apprentissage de cette technique chorégraphique.

Ce façonnage du corps est mis en valeur non seulement par la jupe mais aussi par les sequins, pièces dorées accrochées au foulard. Ces petites pièces, dont un professeur m'explique que les " ghawâzî »" les recevaient de leur public lorsqu'elles dansaient et les accrochaient alors à leur foulard, sont à la fois parure et signe de prospérité, d'abondance. On pense aussi à la monnaie reçue coutumièrement par les mariés. Pour Aurélie, ces petites pièces qui peuvent apparaître comme un détail sont essentielles. Le «vrai» costume de danseuse orientale doit être sonore. Ses parents lui avaient ramené une tenue de danse marocaine qui comportait de grands voiles mais la disposition des sequins, épars sur le tissu,

20. Christophe Apprill affirme ainsi : «La combinaison d'une éducation morale, du traitement de la femme dans la publicité et des protocoles de rééducation en kinésithérapie se traduit par un placement rétroversé du bassin, dont les femmes découvrent avec perplexité le verrouillage » (2008: 54). L'étude de Kaufmann sur les seins nus témoigne de la volonté de certaines femmes de s'approprier leur corps et de s'émanciper d'une éducation réprimant leur sensualité (2010:64).

21. Sur les ghawâzî qui étaient considérées comme des «tziganes du Moyen-Orient », cf. Henni-Chebra et Poché (dir.) 1996. 
ne les faisaient pas cliqueter : «Je ne voyais pas l'intérêt, ça faisait pas de bruit : c'était juste des morceaux de tissus avec des disques un peu éparpillés partout. » Elle se fait alors refaire une tenue sur mesure par sa tante qui est couturière. Elle prend soin qu'y soient accrochés des sequins bruissants autour de la poitrine et autour de la taille. À la danse orientale, les femmes exposent ainsi sans retenue leurs attraits charnels. Mis en valeur visuellement par les mouvements ondulatoires qui les « isolent» et auditivement par le bruit que provoque la danse, lieu de la danse et instrument de musique, ces parties du corps deviennent ainsi la « richesse féminine ». Parure (ici objets précieux de petite taille ornant les vêtements), son, danse et façonnage corporel sont indissociables. Les parures-pièces, symbole de prospérité, célèbrent à la fois les pouvoirs féminins de séduction et génésiques. Tout comme le foulard qui, indissociablement magnifie le bassin et est nécessaire à la bonne exécution de la danse, il faut qu'elles sonnent lors de la danse. On peut estimer, en suivant les propos de Danièle Cohn pour qui « l'ornement est un cas particulier de la métaphorisation » (voir Cohn 2008 : 87-93), un « plaisir de reconnaissance à l'articulation de l'action et de la cognition », qu'elles sont constitutives de la beauté de la danseuse.

\section{De l'efficacité esthétique}

Bien qu'il n'y ait apparemment aucune prescription obligatoire, les différents styles chorégraphiques proposés dans les écoles de danse amateur ont une fonction sociale importante : façonner les corps féminins selon certaines images, leur conférer et faire ressortir certaines qualités en fonction des âges de la vie. Si la danse classique identifie les jeunes filles à la beauté paradoxale de la ballerine, la liberté gestuelle inhérente à la danse contemporaine, s'apparente davantage à une pure expressivité corporelle, d'une part, et les danses étrangères de l'autre, «font le couple » ou/et magnifient leurs pouvoirs sexuels et de fécondité au point qu'on peut dire que ces danses « font» littéralement les femmes qui s'y investissent. Aussi la place des danses étrangères dans ce parcours est-elle éclairante : si les femmes passent par elles pour s'exprimer c'est aussi que, je pense l'avoir montré ci-dessus, la féminité en tant que telle (le désir qu'elle suscite, sa capacité à « fabriquer » de l'humain...) est pour les femmes elles-mêmes une énigme (voir aussi Kofman 1980) et constitue un des paradigmes de l'altérité, mais aussi une valeur qu'il m'apparaît important de préserver.

L'analyse de la pratique de ces différents styles chorégraphiques permet de saisir qu'elle est d'abord l'apprentissage d'un langage esthétique et émotionnel précis. La pratique amateur témoigne ainsi de l'efficacité des techniques corporelles : les gestuelles chorégraphiques qui sont ornement, parure incorporée, participent tout comme les costumes d' " un art d'utiliser le corps humain efficace esthétiquement » pour reprendre l'expression employée par Marc Leveratto relisant la conférence prononcée par Marcel Mauss sur les techniques du corps (Leveratto 2006). Ces pratiques tendent donc à façonner esthétiquement les corps féminins mais aussi, à séparer le masculin du féminin afin qu'ils se rejoignent dans une union féconde. La perception esthétique consiste en effet, selon Véronique Nahoum-Grappe, en une « étreinte mimée $»^{22}$. C'est là, écrivait-elle, une des fonctions de la beauté.

22. Je reprends ici la notion forgée par Véronique Nahoum-Grappe qui entend par là la transposition de la stratégie désirante dans l'acte perceptif (1988: 5-6). 


\section{Références bibliographiques}

Apprill, Christophe, 2008. Tango le couple, le bal et la scène. Paris : Autrement.

Boukновza, Noria, 2002. Les Femmes dans l'ombre du jour. Castenaud-la-Chapelle : Hydre éditions.

Bulletin du département des études et de la prospective du Ministère de la Communication et de la Culture, 112, juin 1996.

Calderoli, Lidia, 1993. «Marquages permanents du corps en Afrique subsaharienne : le processus technique comme signifiant », L'Ethnographie, $114: 105-128$.

Ciambelli, Patrizia, 2002. Bijoux à secrets. Paris : Édition de la Maison des sciences de l'homme.

Cohn, Danièle, 2008. Posteface à Karl Philipp, Moritz, Sur l'ornement. Paris : Éditions rue d'Ulm-Presses de l'Ecole Normale Supérieur-Musée du quai Branly, Æsthetica.

Eco, Umberto, 2007. Histoire de la laideur, trad. Myriem Bouzaher. Paris : Flammarion.

FABRE, Daniel, 1986. « La voie des oiseaux. Sur quelques récits d'apprentissage », L’Homme, 99 : 7-40.

Faure, Sylvia, 2000. Apprendre par corps. Socio-anthropologie des techniques de danse. Paris : La dispute.

Foucault, Michel, 1975. Surveiller et punir. Paris : Gallimard.

Henni-Chebra, Djamila et Christian Poche (dir.). 1996. Les Danses dans le monde arabe ou l'héritage des almées. Paris : l'Harmattan.

Houseman Michael, 1986. « Le mal pour le mâle », in J. Hainard et R Kaehr (éds), Le Mal et la Douleur. Neuchâtel : Musée d'ethnographie, 89-99.

Jeudy-Ballini, Monique, 1999. « Dédommager le désir : le prix de l'émotion en Nouvelle Bretagne », Terrain, $32: 5-20$.

Kaufmann, Jean-Claude, 1993. Sociologie du couple. Paris : PUF.

--_-, 2010. Corps de femmes, regards d'hommes. Sociologie des seins nus. Paris : Nathan.

Kofman, Sarah, 1980. L'Énigme de la femme. Paris : Galilée.

Leblon Bernard, 1995. Flamenco. Arles : Actes Sud.

Legendre, Pierre, 1978. La passion d'être un autre. Paris : Seuil.

Leroi-Gourhan, André. 1964. Le Geste et la Parole, t.2, La Mémoire et les Rythmes. Paris : Albin Michel.

Leveratto, Jean-Marc, 2006, «Lire Mauss, l'authentification des techniques du corps et ses enjeux épistémologiques », Le Portique, 17, en ligne http://leportique.revues.org/index778.html

Nahoum-Grappe, Véronique, 1988. «Beauté et laideur; histoire et anthropologie de la forme humaine », Chimères, 5/6:1-27.

1996. Le Féminin. Paris : Hachette.

NANCY, Jean-Luc, 2009. La Beauté. Paris : Bayard.

Nicolas, Maud, 2000. « Ce que danser veut dire », Terrain, $35: 41-56$.

Pasqualino, Caterina, 1998. Dire le chant. Les gitans flamencos d'Andalousie. Paris : CNRS éditions-Éditions de la Maison des Sciences de l'Homme.

Rannou, Jeanine et Ionela Roharik, 2006. Les danseurs. Un métier d'engagement. Paris : La Documentation française.

Turner Victor, 1991. The Ritual Process, Structure and antistructures. Ithaca (N.Y.) : Cornell Paperbacks.

VALEnTin, Virginie, 2000. « L'acte blanc ou le passage impossible. Les paradoxes de la danse classique », Terrain, $35: 95-108$. 
----, 2005. De fille en aiguille. Figures du féminin et façonnage du corps dans la danse classique, thèse de doctorat en anthropologie sociale et historique, université de Toulouse-le Mirail.

Van Gennep, Arnold, 1909. Les Rites de Passage. Paris : Picard.

Verdier, Yvonne, 1979. Façons de dire, façons de faire. La laveuse, la couturière, la cuisinière. Paris : Gallimard, Bibliothèque des sciences humaines.

----, 1995. Coutume et destin, Thomas Hardy et autres essais. Paris : Gallimard, Bibliothèque des sciences humaines.

Vigarello, Georges, 1978. Le Corps redressé, histoire d'un pouvoir pédagogique. Paris : Delage. 